\title{
ON THE MAXIMUM EXCEEDANCE OF A SEQUENCE OF RANDOM VARIABLES OVER A RENEWAL THRESHOLD
}

\author{
XUEMIAO $\mathrm{HAO}^{* * *}$ AND \\ QIHE TANG, ${ }^{* * * *}$ The University of Iowa \\ LI WEI, ${ }^{* * * *}$ Renmin University of China
}

\begin{abstract}
In this paper we study the tail behavior of the maximum exceedance of a sequence of independent and identically distributed random variables over a random walk. For both light-tailed and heavy-tailed cases, we derive a precise asymptotic formula, which extends and unifies some existing results in the recent literature of applied probability.
\end{abstract}

Keywords: Asymptotics; exceedance; random walk; tail probability; the classes $\mathcal{L}(\gamma)$ and $\delta(\gamma)$

2000 Mathematics Subject Classification: Primary 60G50

Secondary $60 \mathrm{G} 70$

\section{Introduction and main result}

Let $\left\{Y_{n}, n=1,2, \ldots\right\}$ be a sequence of independent and identically distributed (i.i.d.) random variables with generic random variable $Y$, common distribution $F=1-\bar{F}$ on $(-\infty, \infty)$, and $0<v_{F}=\int_{0}^{\infty} \frac{\bar{F}}{F}(u) \mathrm{d} u<\infty$. Define the equilibrium distribution of $F$ as

$$
F_{\mathrm{e}}(x)=\frac{1}{v_{F}} \int_{0}^{x} \bar{F}(u) \mathrm{d} u, \quad x \geq 0 .
$$

For every constant $\mu>0$, the maximum

$$
M_{0}=\sup _{n \geq 1}\left(Y_{n}-(n-1) \mu\right)
$$

is finite almost surely. If $F_{\mathrm{e}}$ is long tailed (i.e. $\left.\lim _{x \rightarrow \infty} \overline{F_{\mathrm{e}}}(x+1) / \overline{F_{\mathrm{e}}}(x)=1\right)$ then it is easy to check that

$$
\lim _{x \rightarrow \infty} \frac{\operatorname{Pr}\left(M_{0}>x\right)}{\int_{x}^{\infty} \bar{F}(u) \mathrm{d} u}=\frac{1}{\mu} .
$$

Motivated by the observation above, in this paper we study the tail probability of the maximum exceedance of the sequence $\left\{Y_{n}, n=1,2, \ldots\right\}$ over a random walk with positive drift. Precisely, let $\left\{\left(X_{n}, Y_{n}\right), n=1,2, \ldots\right\}$ be a sequence of i.i.d. random pairs with generic

Received 27 May 2008; revision received 31 December 2008.

* Postal address: Department of Statistics and Actuarial Science, The University of Iowa, 241 Schaeffer Hall, Iowa City, IA 52242, USA.

** Email address: xuhao@stat.uiowa.edu

*** Email address: qtang@stat.uiowa.edu

**** Postal address: School of Finance, Renmin University of China, Beijing, 100872, P. R. China.

Email address: weil@ruc.edu.cn 
random pair $(X, Y)$. Assume that $\mathrm{E} X=\mu>0$ and that $Y$ follows a distribution $F$ on $(-\infty, \infty)$ with $0<v_{F}<\infty$. Then, the maximum

$$
M=\sup _{n \geq 1}\left(Y_{n}-S_{n-1}\right)
$$

with $S_{n-1}=\sum_{i=1}^{n-1} X_{i}$, is finite almost surely, where a sum over an empty set of indices is equal to 0 by convention.

To state our conditions on $Y$, we introduce the following distribution classes, which are popular in applied probability. A distribution $F$ on $(-\infty, \infty)$ is said to belong to the class $\mathcal{L}(\gamma)$ for some $\gamma \geq 0$ if $\bar{F}(x)>0$ for all $x$ and

$$
\lim _{x \rightarrow \infty} \frac{\bar{F}(x-y)}{\bar{F}(x)}=\mathrm{e}^{\gamma y} \text { for all } y \in(-\infty, \infty) .
$$

Note that $\mathcal{L}(0)$ reduces to the well-known class $\mathcal{L}$ of long-tailed distributions. Furthermore, a distribution $F$ on $[0, \infty)$ is said to belong to the class $\delta(\gamma)$ for some $\gamma \geq 0$ if $F \in \mathcal{L}(\gamma)$ and the limit

$$
\lim _{x \rightarrow \infty} \frac{\overline{F^{2 *}}(x)}{\bar{F}(x)}=2 c
$$

exists and is finite, where $F^{2 *}$ denotes the two-fold convolution of $F$. More generally, a distribution $F$ on $(-\infty, \infty)$ is also said to belong to the class $\delta(\gamma)$ if $F_{+}(x)=F(x) \mathbf{1}_{\{x \geq 0\}}$ does, where $\mathbf{1}_{E}$ denotes the indicator of an event $E$. Note that $\&(0)$ reduces to the well-known class $\&$ of subexponential distributions.

For the sake of consistency, for a random variable $X$ with mean $\mu>0$, we make a convention that

$$
\left.\frac{\gamma}{1-\mathrm{E}^{-\gamma X}}\right|_{\gamma=0}=\frac{1}{\mu} \text {. }
$$

The main result of this paper is given below.

Theorem 1.1. Consider the i.i.d. sequence $\left\{\left(X_{n}, Y_{n}\right), n=1,2, \ldots\right\}$ and the maximum $M$ defined in (1.1), where $\mathrm{E} X=\mu>0$ and $Y$ is distributed by $F$ with $0<v_{F}<\infty$. Then, the relation

$$
\lim _{x \rightarrow \infty} \frac{\operatorname{Pr}(M>x)}{\int_{x}^{\infty} \bar{F}(u) \mathrm{d} u}=\frac{\gamma}{1-\mathrm{E}^{-\gamma X}}
$$

holds under one of the following groups of conditions:

(i) $F_{\mathrm{e}} \in \mathcal{L}(\gamma)$ for some $\gamma \geq 0$, $\mathrm{E} X^{2}<\infty$, and $\mathrm{E}^{-\beta X}<1$ for some $\beta>\gamma$;

(ii) $F_{\mathrm{e}} \in \delta(\gamma)$ for some $\gamma \geq 0, \operatorname{Pr}(-X>x)=o(\bar{F}(x))$, and $\mathrm{E}^{-\gamma X}<1$ provided $\gamma>0$.

Clearly, $\mathrm{E}^{-\alpha X}$, as a function of $\alpha$, is convex over all $\alpha$ for which $\mathrm{E}^{-\alpha X}$ is finite. Hence, for Theorem 1.1(i), $\mathrm{E}^{-\alpha X}<1$ for every $\alpha \in(0, \beta]$.

As shown in Lemma 2.1, below, for every $\gamma \geq 0$, the condition $F_{\mathrm{e}} \in \mathcal{L}(\gamma)$ is equivalent to the relation

$$
\lim _{x \rightarrow \infty} \frac{\bar{F}(x)}{\int_{x}^{\infty} \bar{F}(u) \mathrm{d} u}=\gamma .
$$

In particular, the condition $F_{\mathrm{e}} \in \mathcal{L}$ (or, equivalently, relation (1.4) with $\gamma=0$ ) is fulfilled by most cited heavy-tailed distributions, including all long-tailed or dominatedly varying-tailed 
distributions with finite mean; see Theorem 3.1 and Example 3.1 of Su and Tang (2003) for more details.

Some closely related works are summarized as follows. Robert (2005) considered a special case of our Theorem 1.1(i) with $\gamma=0$ and $X$ positive, and proposed an application to ruin theory in the presence of dividends paid out at a sequence of random epochs. Araman and Glynn (2006) systematically studied the same problem in the framework of a perturbed random walk for various cases. Their Theorem 3 corresponds to a special case of our Theorem 1.1(i) with $\gamma>0, X, Y$ independent, and $F$ exponential, but under a slightly weaker moment condition on $X$ than ours. Their Theorem 4, assuming that $F$ has a continuous hazard rate function converging to 0 , corresponds to a special case of our Theorem 1.1(i) with $\gamma=0$ and $X, Y$ independent. Palmowski and Zwart (2007) also studied the same problem but in the framework of a regenerative process. In terms of their model in which the regenerative process $\{S(t), t \geq 0\}$ has renewal epochs $0=T_{0}<T_{1}<\cdots$, the random variables $X_{n}$ and $Y_{n}$ in our theorems correspond to $S\left(T_{n-1}\right)-S\left(T_{n}\right)$ and $\sup _{T_{n-1} \leq t<T_{n}} S(t)-S\left(T_{n-1}\right)$, respectively. In particular, their Theorem 1 corresponds to our Theorem 1.1(ii) with $\gamma=0$ under the assumption that the equilibrium distribution of $(-X) \vee Y$ is subexponential, and their Theorem 2 corresponds to our Theorem 1.1 with $\gamma>0$ under slightly more general conditions than ours.

In the rest of this paper, after preparing a series of lemmas in Section 2, we prove cases (i) and (ii) of Theorem 1.1 in Sections 3 and 4, respectively.

\section{Lemmas}

Throughout this paper, all limit relationships are for $x \rightarrow \infty$ unless stated otherwise. For two positive functions $a(\cdot)$ and $b(\cdot)$, we write $a(x) \sim b(x)$ if $\lim a(x) / b(x)=1, a(x) \lesssim b(x)$ if $\lim \sup a(x) / b(x) \leq 1$, and $a(x) \gtrsim b(x)$ if $\liminf a(x) / b(x) \geq 1$.

Lemma 2.1. Assume that $F$ on $(-\infty, \infty)$ satisfies $0<\int_{0}^{\infty} \bar{F}(u) \mathrm{d} u<\infty$. For each $\gamma \geq 0$, $F_{\mathrm{e}} \in \mathcal{L}(\gamma)$ if and only if relation (1.4) holds.

Proof. For $\gamma>0$, see Lemma 3.1 of Tang (2007). For $\gamma=0$, observe that

$$
0 \leq \frac{\overline{F_{\mathrm{e}}}(x)-\overline{F_{\mathrm{e}}}(x+1)}{\overline{F_{\mathrm{e}}}(x)} \leq \frac{\bar{F}(x)}{\int_{x}^{\infty} \bar{F}(u) \mathrm{d} u} \leq \frac{\overline{F_{\mathrm{e}}}(x-1)-\overline{F_{\mathrm{e}}}(x)}{\overline{F_{\mathrm{e}}}(x)},
$$

from which the desired equivalence follows.

Lemma 2.2. Let $\left\{\xi_{n}, n=1,2, \ldots\right\}$ be a sequence of i.i.d. random variables with generic random variable $\xi$ satisfying $-\infty<\mathrm{E} \xi<0$ and $\operatorname{Pr}(\xi>0)>0$. Then, $\mathrm{E}(\xi \vee 0)^{2}<\infty$ if and only if

$$
\sum_{n=1}^{\infty} \operatorname{Pr}\left(\sum_{i=1}^{n} \xi_{i}>0\right)<\infty .
$$

Proof. See Lemma 3.1 of Robert (2005).

Lemma 2.3. If $F \in \mathcal{L}(\gamma)$ for some $\gamma \geq 0$ then, for every $\beta>\gamma$,

(i) there exist some positive constants $c_{0}$ and $x_{0}$ such that, for all $x \geq y \geq x_{0}$,

$$
\frac{\bar{F}(y)}{\bar{F}(x)} \leq c_{0} \mathrm{e}^{\beta(x-y)}
$$

(ii) $\mathrm{e}^{-\beta x}=o(\bar{F}(x))$. 
Proof. (i) Note that $F \in \mathcal{L}(\gamma)$ if and only if $\bar{F}(\ln x)$ is regularly varying of index $-\gamma$. Thus, the desired conclusion is a straightforward consequence of the well-known Potter's bound; see Theorem 1.5.6(iii) of Bingham et al. (1987).

(ii) For some $\beta^{\prime}, \gamma<\beta^{\prime}<\beta$, by item (i), there exist some positive constants $c_{0}$ and $x_{0}$ such that, for all $x \geq x_{0}$,

$$
\frac{\bar{F}\left(x_{0}\right)}{\bar{F}(x)} \leq c_{0} \exp \left(\beta^{\prime}\left(x-x_{0}\right)\right) .
$$

Hence, the relation $\mathrm{e}^{-\beta x}=o(\bar{F}(x))$ holds.

Lemma 2.4. Let $F, G, G_{1}$, and $G_{2}$ be distributions on $(-\infty, \infty)$.

(i) If $F \in \mathcal{L}(\gamma)$ for some $\gamma \geq 0$ and $\int_{-\infty}^{\infty} \mathrm{e}^{\beta u} G(\mathrm{~d} u)<\infty$ for some $\beta>\gamma$, then

$$
\lim _{x \rightarrow \infty} \frac{\overline{F * G}(x)}{\bar{F}(x)}=\int_{-\infty}^{\infty} \mathrm{e}^{\gamma u} G(\mathrm{~d} u) .
$$

(ii) If $F \in s(\gamma)$ for some $\gamma \geq 0$ and the limit $c_{i}=\lim \overline{G_{i}}(x) / \bar{F}(x)$ exists and belongs to $[0, \infty)$ for $i=1,2$, then

$$
\lim _{x \rightarrow \infty} \frac{\overline{G_{1} * G_{2}}(x)}{\bar{F}(x)}=c_{1} \int_{-\infty}^{\infty} \mathrm{e}^{\gamma u} G_{2}(\mathrm{~d} u)+c_{2} \int_{-\infty}^{\infty} \mathrm{e}^{\gamma u} G_{1}(\mathrm{~d} u) .
$$

Proof. (i) See Lemma 2.1 of Pakes (2004). Note that, under the current conditions, the relation $\bar{G}(x)=o(\bar{F}(x))$, as required in Lemma 2.1 of Pakes (2004), holds automatically by Lemma 2.3(ii).

(ii) See Proposition 2 of Rogozin and Sgibnev (1999).

Lemma 2.5. Let $\left\{\xi_{n}, n=1,2, \ldots\right\}$ be a sequence of i.i.d. random variables with finite mean $\mu$. Then, for arbitrarily small $\varepsilon, \delta>0$, there exists some constant $C>0$ such that

$$
\operatorname{Pr}\left(\bigcap_{n=1}^{\infty}\left(n(\mu-\delta)-C \leq \sum_{i=1}^{n} \xi_{i} \leq n(\mu+\delta)+C\right)\right)>1-\varepsilon .
$$

Proof. The proof follows from the proof of Lemma 3.1 of Asmussen et al. (1999) with some obvious modifications.

Lemma 2.6. Assume that $F_{\mathrm{e}} \in \delta(\gamma)$ for some $\gamma \geq 0$. Let $\left\{\xi_{n}, n=1,2, \ldots\right\}$ be a sequence of i.i.d. random variables with generic random variable $\xi$ satisfying $-\infty<\mathrm{E} \xi<0, \operatorname{Pr}(\xi>$ $x)=o(\bar{F}(x))$, and $\mathrm{E}^{\gamma \xi}<1$ provided $\gamma>0$. Then,

$$
\operatorname{Pr}\left(\sup _{n \geq 0} \sum_{i=1}^{n} \xi_{i}>x\right)=o\left(\overline{F_{\mathrm{e}}}(x)\right) .
$$

Proof. For arbitrarily fixed $N$, we have

$$
\begin{aligned}
\operatorname{Pr}\left(\sup _{n \geq 0} \sum_{i=1}^{n} \xi_{i}>x\right) & \leq \operatorname{Pr}\left(\sup _{0 \leq n \leq N} \sum_{i=1}^{n} \xi_{i}>x\right)+\operatorname{Pr}\left(\sum_{i=1}^{N} \xi_{i}+\sup _{n \geq N} \sum_{i=N+1}^{n} \xi_{i}>x\right) \\
& =I_{1}(x, N)+I_{2}(x, N) .
\end{aligned}
$$


By Lemma 2.1, $\operatorname{Pr}(\xi>x)=o(\bar{F}(x))=o\left(\overline{F_{\mathrm{e}}}(x)\right)$. Then, by Lemma 2.4(ii),

$$
I_{1}(x, N) \leq \operatorname{Pr}\left(\sum_{i=1}^{N}\left(\xi_{i} \vee 0\right)>x\right)=o\left(\overline{F_{\mathrm{e}}}(x)\right) .
$$

To consider $I_{2}(x, N)$, for arbitrarily small $\varepsilon>0$, introduce a random variable $\eta$ satisfying

$$
\operatorname{Pr}(\eta>x)=\operatorname{Pr}(\xi>x) \vee \varepsilon \bar{F}(x) .
$$

Clearly, $\operatorname{Pr}(\eta>x) \sim \varepsilon \bar{F}(x)$. Since $\eta=\eta(\varepsilon)$ converges to $\xi$ in distribution as $\varepsilon \searrow 0$ for all small $\varepsilon>0$, we have $\mathrm{E} \eta<0$ and $\mathrm{Ee}^{\gamma \eta}<1$ provided $\gamma>0$. Let $\left\{\eta_{n}, n=1,2, \ldots\right\}$ be a sequence of i.i.d. copies of $\eta$ independent of $\left\{\xi_{n}, n=1,2, \ldots\right\}$. By Theorem 2 of Veraverbeke (1977), it holds, for some constant $c(\gamma, \varepsilon)>0$, that

$$
\operatorname{Pr}\left(\sup _{n \geq N} \sum_{i=N+1}^{n} \eta_{i}>x\right)=\operatorname{Pr}\left(\sup _{n \geq 0} \sum_{i=1}^{n} \eta_{i}>x\right) \sim c(\gamma, \varepsilon) \overline{F_{\mathrm{e}}}(x) .
$$

When $\gamma>0$, the expression of $c(\gamma, \varepsilon)$ is rather involved. However, when $\gamma=0$, we have the transparent expression $c(0, \varepsilon)=-\varepsilon v_{F} / \mathrm{E} \eta$. Then, by Lemma 2.4(ii),

$$
I_{2}(x, N) \leq \operatorname{Pr}\left(\sum_{i=1}^{N} \xi_{i}+\sup _{n \geq N} \sum_{i=N+1}^{n} \eta_{i}>x\right) \sim\left(\mathrm{E}^{\gamma \xi}\right)^{N} c(\gamma, \varepsilon) \overline{F_{\mathrm{e}}}(x) .
$$

Substituting (2.3) and (2.4) into (2.2) yields

$$
\limsup _{x \rightarrow \infty} \frac{1}{\overline{F_{\mathrm{e}}}(x)} \operatorname{Pr}\left(\sup _{n \geq 0} \sum_{i=1}^{n} \xi_{i}>x\right) \leq\left(\mathrm{Ee}^{\gamma \xi}\right)^{N} c(\gamma, \varepsilon) .
$$

If $\gamma>0$ with $\varepsilon$ fixed, we let $N \rightarrow \infty$, while if $\gamma=0$, we let $\varepsilon \searrow 0$. Thus, in any case, the right-hand side of the above goes to 0 and the proof is complete.

\section{Proof of Theorem 1.1(i)}

\subsection{Preliminary results}

Proposition 3.1. Under the conditions of Theorem 1.1(i), it holds, for arbitrarily small $\varepsilon>0$, all $0<\delta<1$, and all large $k$, that

$$
\sum_{n=k+1}^{\infty} \operatorname{Pr}\left(Y_{n}-S_{n-1}>x, S_{n-1}<(n-1) \mu(1-\delta)\right) \lesssim \varepsilon \int_{x}^{\infty} \bar{F}(u) \mathrm{d} u .
$$

Proof. Let $0<\delta<1$ and $D>0$ be arbitrarily fixed. For all $x>D$, according to the range of $S_{n-1}$ we split the left-hand side of (3.1) into three parts as

$$
\begin{gathered}
\sum_{n=k+1}^{\infty} \operatorname{Pr}\left(Y_{n}-S_{n-1}>x, S_{n-1} \in(0,(n-1) \mu(1-\delta))\right. \\
\cup(-x+D, 0] \cup(-\infty,-x+D]) \\
=J_{1}(x, k, \delta)+J_{2}(x, k, D)+J_{3}(x, k, D)
\end{gathered}
$$


Using Lemmas 2.1 and 2.2, for arbitrarily small $\varepsilon>0$ and all large $k$,

$$
J_{1}(x, k, \delta) \leq \bar{F}(x) \sum_{n=k+1}^{\infty} \operatorname{Pr}\left(\sum_{i=1}^{n-1}\left(\mu(1-\delta)-X_{i}\right)>0\right) \lesssim \frac{\varepsilon}{2} \int_{x}^{\infty} \bar{F}(u) \mathrm{d} u .
$$

Furthermore, by Lemma 2.3(i), there exist some constants $c_{0}, D>0$ such that, for all $x \geq$ $x+y-1 \geq D-1$ and all large $k$,

$$
\begin{aligned}
J_{2}(x, k, D) & =\sum_{n=k+1}^{\infty} \int_{-x+D}^{0} \bar{F}(x+y) \operatorname{Pr}\left(S_{n-1} \in \mathrm{d} y\right) \\
& \leq \sum_{n=k+1}^{\infty} \int_{-x+D}^{0}\left(\int_{x+y-1}^{x+y} \bar{F}(u) \mathrm{d} u\right) \operatorname{Pr}\left(S_{n-1} \in \mathrm{d} y\right) \\
& \leq \int_{x}^{\infty} \bar{F}(u) \mathrm{d} u \sum_{n=k+1}^{\infty} \int_{-x+D}^{0} \frac{\overline{F_{\mathrm{e}}}(x+y-1)}{\overline{F_{\mathrm{e}}}(x)} \operatorname{Pr}\left(S_{n-1} \in \mathrm{d} y\right) \\
& \leq c_{0} \int_{x}^{\infty} \bar{F}(u) \mathrm{d} u \sum_{n=k+1}^{\infty} \operatorname{Eexp}\left(-\beta\left(S_{n-1}-1\right)\right) \\
& \leq \frac{\varepsilon}{2} \int_{x}^{\infty} \bar{F}(u) \mathrm{d} u .
\end{aligned}
$$

For $D$ specified in (3.4) and all $k$, employ Markov's inequality and Lemma 2.3(ii) to obtain

$$
J_{3}(x, k, D) \leq \sum_{n=k+1}^{\infty} \operatorname{Pr}\left(S_{n-1} \leq-x+D\right) \leq \sum_{n=k+1}^{\infty} \frac{\mathrm{E} \exp \left(-\beta S_{n-1}\right)}{\mathrm{e}^{\beta(x-D)}}=o\left(\overline{F_{\mathrm{e}}}(x)\right) .
$$

Substituting (3.3)-(3.5) into (3.2) yields (3.1).

Proposition 3.2. Under the conditions of Theorem 1.1( $i$ ), it holds, for each $k=2,3, \ldots$, that

$$
\sum_{1 \leq n<m \leq k} \operatorname{Pr}\left(Y_{n}-S_{n-1}>x, Y_{m}-S_{m-1}>x\right)=o\left(\overline{F_{\mathrm{e}}}(x)\right) .
$$

Proof. Let $\delta>0$ be a constant satisfying $\beta(1-\delta)>\gamma$. For $1=n<m \leq k$,

$$
\begin{aligned}
\operatorname{Pr}\left(Y_{1}\right. & \left.>x, Y_{m}-S_{m-1}>x\right) \\
& \leq \operatorname{Pr}\left(-S_{m-1}>(1-\delta) x\right)+\operatorname{Pr}\left(Y_{1}>x, Y_{m}-S_{m-1}>x,-S_{m-1} \leq(1-\delta) x\right) \\
& \leq \mathrm{e}^{-\beta(1-\delta) x} \operatorname{E} \exp \left(-\beta S_{m-1}\right)+\operatorname{Pr}\left(Y_{1}>x, Y_{m}>\delta x\right) \\
& =o\left(\overline{F_{\mathrm{e}}}(x)\right),
\end{aligned}
$$

where we used Markov's inequality and Lemmas 2.1 and 2.3(ii). Similarly, for $1<n<m \leq k$,

$$
\begin{aligned}
\operatorname{Pr}\left(Y_{n}-\right. & \left.S_{n-1}>x, Y_{m}-S_{m-1}>x\right) \\
\leq & \operatorname{Pr}\left(-S_{n-1}>(1-\delta) x\right) \\
& \quad+\operatorname{Pr}\left(Y_{n}-S_{n-1}>x, Y_{m}-S_{m-1}>x,-S_{n-1} \leq(1-\delta) x\right) \\
\leq & \mathrm{e}^{-\beta(1-\delta) x} \operatorname{E} \exp \left(-\beta S_{n-1}\right) \\
& +\int_{-\infty}^{(1-\delta) x} \operatorname{Pr}\left(Y_{n}>x-y, Y_{m}-S_{n, m-1}>x-y\right) \operatorname{Pr}\left(-S_{n-1} \in \mathrm{d} y\right),
\end{aligned}
$$


where $S_{n, m-1}=\sum_{i=n}^{m-1} X_{i}$. By (3.7), it holds uniformly for all $y \leq(1-\delta) x$ that

$$
\operatorname{Pr}\left(Y_{n}>x-y, Y_{m}-S_{n, m-1}>x-y\right)=o(1) \overline{F_{\mathrm{e}}}(x-y) .
$$

Hence, by Lemmas 2.3(ii) and 2.4(i),

$$
\begin{aligned}
\operatorname{Pr}\left(Y_{n}\right. & \left.-S_{n-1}>x, Y_{m}-S_{m-1}>x\right) \\
& =o\left(\overline{F_{\mathrm{e}}}(x)\right)+o(1) \int_{-\infty}^{(1-\delta) x} \overline{F_{\mathrm{e}}}(x-y) \operatorname{Pr}\left(-S_{n-1} \in \mathrm{d} y\right) \\
& =o\left(\overline{F_{\mathrm{e}}}(x)\right) .
\end{aligned}
$$

A combination of (3.7) and (3.8) gives (3.6).

\subsection{Proof of Theorem 1.1(i) for $\gamma>0$}

We first prove the asymptotic upper bound. For some $0<\delta<1$ and each $k=1,2, \ldots$,

$$
\begin{aligned}
\operatorname{Pr}(M>x) \leq & \left(\sum_{n=1}^{k}+\sum_{n=k+1}^{\infty}\right) \operatorname{Pr}\left(Y_{n}-S_{n-1}>x\right) \\
\leq & \sum_{n=1}^{k} \operatorname{Pr}\left(Y_{n}-S_{n-1}>x\right)+\sum_{n=k+1}^{\infty} \bar{F}(x+(n-1) \mu(1-\delta)) \\
& +\sum_{n=k+1}^{\infty} \operatorname{Pr}\left(Y_{n}-S_{n-1}>x, S_{n-1}<(n-1) \mu(1-\delta)\right) \\
= & K_{1}(x, k)+K_{2}(x, k, \delta)+K_{3}(x, k, \delta) .
\end{aligned}
$$

By Proposition 3.1, it holds, for arbitrarily small $\varepsilon>0$ and all large $k$, that

$$
K_{3}(x, k, \delta) \lesssim \frac{\varepsilon}{2} \int_{x}^{\infty} \bar{F}(u) \mathrm{d} u .
$$

Since $F_{\mathrm{e}} \in \mathcal{L}(\gamma)$, it holds, for all large $k$, that

$$
K_{2}(x, k, \delta) \leq \frac{1}{\mu(1-\delta)} \int_{x+(k-1) \mu(1-\delta)}^{\infty} \bar{F}(u) \mathrm{d} u \lesssim \frac{\varepsilon}{2} \int_{x}^{\infty} \bar{F}(u) \mathrm{d} u .
$$

With $k$ specified in (3.10) and (3.11), by Lemma 2.4(i) and relation (1.4), we have

$$
K_{1}(x, k) \sim \bar{F}(x) \sum_{n=1}^{k} \mathrm{E} \exp \left(-\gamma S_{n-1}\right) \lesssim \frac{\gamma}{1-\mathrm{Ee}^{-\gamma X}} \int_{x}^{\infty} \bar{F}(u) \mathrm{d} u
$$

Substituting (3.10)-(3.12) into (3.9) and using the arbitrariness of $\varepsilon>0$, we obtain

$$
\limsup _{x \rightarrow \infty} \frac{\operatorname{Pr}(M>x)}{\int_{x}^{\infty} \bar{F}(u) \mathrm{d} u} \leq \frac{\gamma}{1-\mathrm{E}^{-\gamma X}}
$$


Next, we turn to the proof of the asymptotic lower bound. Obviously, for each $k=1,2, \ldots$, using Bonferroni's inequality,

$$
\begin{aligned}
\operatorname{Pr}(M>x) & \geq \operatorname{Pr}\left(\bigcup_{n=1}^{k}\left(Y_{n}-S_{n-1}>x\right)\right) \\
& \geq K_{1}(x, k)-\sum_{1 \leq n<m \leq k} \operatorname{Pr}\left(Y_{n}-S_{n-1}>x, Y_{m}-S_{m-1}>x\right),
\end{aligned}
$$

where $K_{1}(x, k)$ is the same as in (3.9). Similar to (3.12), for arbitrarily small $\varepsilon>0$ and all large $k$,

$$
K_{1}(x, k) \gtrsim(1-\varepsilon) \frac{\gamma}{1-\mathrm{E} \mathrm{e}^{-\gamma X}} \int_{x}^{\infty} \bar{F}(u) \mathrm{d} u .
$$

By Proposition 3.2, relation (3.6) holds. Substituting (3.6) and (3.14) into (3.13) and using the arbitrariness of $\varepsilon>0$, we have

$$
\liminf _{x \rightarrow \infty} \frac{\operatorname{Pr}(M>x)}{\int_{x}^{\infty} \bar{F}(u) \mathrm{d} u} \geq \frac{\gamma}{1-\mathrm{E}^{-\gamma X}}
$$

\subsection{Proof of Theorem 1.1(i) for $\gamma=0$}

For $\gamma=0$, relation (1.3) becomes

$$
\lim _{x \rightarrow \infty} \frac{\operatorname{Pr}(M>x)}{\int_{x}^{\infty} \bar{F}(u) \mathrm{d} u}=\frac{1}{\mu} .
$$

To derive the asymptotic upper bound, we still use (3.9). By Proposition 3.1, relation (3.10) holds for arbitrarily small $\varepsilon, \delta>0$ and all large $k$. With $k$ specified in (3.10), by $F_{\mathrm{e}} \in \mathcal{L}$ we have

$$
K_{2}(x, k, \delta) \leq \frac{1}{\mu(1-\delta)} \int_{x+(k-1) \mu(1-\delta)}^{\infty} \bar{F}(u) \mathrm{d} u \sim \frac{1}{\mu(1-\delta)} \int_{x}^{\infty} \bar{F}(u) \mathrm{d} u,
$$

while, by Lemmas 2.1 and 2.4(i), it is easy to see that

$$
K_{1}(x, k)=o(1) \int_{x}^{\infty} \bar{F}(u) \mathrm{d} u
$$

Substituting (3.10), (3.16), and (3.17) into (3.9) and using the arbitrariness of $\varepsilon, \delta>0$, we have

$$
\limsup _{x \rightarrow \infty} \frac{\operatorname{Pr}(M>x)}{\int_{x}^{\infty} \bar{F}(u) \mathrm{d} u} \leq \frac{1}{\mu} .
$$

Next, we consider the asymptotic lower bound. For arbitrarily small $\varepsilon, \delta>0$, by Lemma 2.5 , there exists some constant $C>0$ such that inequality (2.1) holds. Write $E_{n}=\{n(\mu-\delta)-C \leq$ $\left.S_{n} \leq n(\mu+\delta)+C\right\}$ for $n=0,1, \ldots$. Then, by Bonferroni's inequality again,

$$
\begin{aligned}
\operatorname{Pr}(M>x) \geq & \operatorname{Pr}\left(\bigcup_{n=1}^{\infty}\left(\left(Y_{n}-S_{n-1}>x\right) \cap E_{n-1}\right)\right) \\
\geq & \sum_{n=1}^{\infty} \operatorname{Pr}\left(\left(Y_{n}-S_{n-1}>x\right) \cap E_{n-1}\right) \\
& \quad-\sum_{1 \leq n<m<\infty} \operatorname{Pr}\left(\left(Y_{n}-S_{n-1}>x\right) \cap\left(Y_{m}-S_{m-1}>x\right) \cap E_{n-1} \cap E_{m-1}\right)
\end{aligned}
$$




$$
\begin{aligned}
& \geq(1-\varepsilon) \sum_{n=1}^{\infty} \bar{F}(x+(n-1)(\mu+\delta)+C) \\
& \quad-\sum_{1 \leq n<m<\infty} \bar{F}(x+(n-1)(\mu-\delta)-C) \bar{F}(x+(m-1)(\mu-\delta)-C) \\
& \geq \frac{1-\varepsilon}{\mu+\delta} \int_{x+C}^{\infty} \bar{F}(u) \mathrm{d} u-\left(\frac{1}{\mu-\delta} \int_{x-(\mu-\delta)-C}^{\infty} \bar{F}(u) \mathrm{d} u\right)^{2} .
\end{aligned}
$$

Since $F_{\mathrm{e}} \in \mathcal{L}$, by the arbitrariness of $\varepsilon, \delta>0$, it follows that

$$
\liminf _{x \rightarrow \infty} \frac{\operatorname{Pr}(M>x)}{\int_{x}^{\infty} \bar{F}(u) \mathrm{d} u} \geq \frac{1}{\mu}
$$

\section{Proof of Theorem 1.1(ii)}

\subsection{Preliminary results}

We respectively establish the counterparts of Propositions 3.1 and 3.2 for the case in which $\gamma>0$.

Proposition 4.1. Under the conditions of Theorem 1.1(ii) for the case in which $\gamma>0$, relation (3.1) holds for arbitrarily small $\varepsilon>0,0<\delta<1$ arbitrarily close to 1, and all large $k$.

Proof. For $0<\delta, d<1$, introduce the maximum $M_{\delta}=\sup _{n \geq 1} \sum_{i=1}^{n-1}\left(\mu(1-d \delta)-X_{i}\right)$, which is finite almost surely. For every $n \geq k+1$, we derive

$$
S_{n-1}=(n-1) \mu(1-d \delta)-\sum_{i=1}^{n-1}\left(\mu(1-d \delta)-X_{i}\right) \geq(n-1) \mu(1-d \delta)-M_{\delta} .
$$

Introduce another random variable $M_{\delta}^{*}$ which is identically distributed as $M_{\delta}$ and is independent of $\left\{Y_{n}, n=1,2, \ldots\right\}$. Therefore, for every $k \geq 1$,

$$
\begin{aligned}
& \sum_{n=k+1}^{\infty} \operatorname{Pr}\left(Y_{n}-S_{n-1}>x, S_{n-1}<(n-1) \mu(1-\delta)\right) \\
& \quad \leq \sum_{n=k+1}^{\infty} \operatorname{Pr}\left(Y_{n}-(n-1) \mu(1-d \delta)+M_{\delta}^{*}>x, M_{\delta}^{*}>k \mu(1-d) \delta\right) \\
& \quad=\int_{k \mu(1-d) \delta}^{\infty} \sum_{n=k+1}^{\infty} \bar{F}(x-y+(n-1) \mu(1-d \delta)) \operatorname{Pr}\left(M_{\delta} \in \mathrm{d} y\right) \\
& \quad \leq \frac{1}{\mu(1-d \delta)}\left(\int_{k \mu(1-d) \delta}^{x}+\int_{x}^{\infty}\right)\left(\int_{x-y}^{\infty} \bar{F}(u) \mathrm{d} u\right) \operatorname{Pr}\left(M_{\delta} \in \mathrm{d} y\right) \\
& \leq \frac{1}{\mu(1-d \delta)}\left(v_{F} \int_{k \mu(1-d) \delta}^{x} \overline{F_{\mathrm{e}}}(x-y)+\int_{x}^{\infty}\left(y-x+v_{F}\right)\right) \operatorname{Pr}\left(M_{\delta} \in \mathrm{d} y\right)
\end{aligned}
$$

To apply Lemma 2.6, we need to choose $\delta$ and $d$ close to 1 such that $\mathrm{Ee}^{\gamma(\mu(1-d \delta)-X)}<1$. Let $F^{*}$ be a distribution defined as $F^{*}(x)=F(x-\mu(1-d \delta))$. Then, $\operatorname{Pr}(\mu(1-d \delta)-X>x)=$ $o\left(\overline{F^{*}}(x)\right)$ and $F_{\mathrm{e}}^{*} \in \delta(\gamma)$. By Lemma 2.6 we have

$$
\operatorname{Pr}\left(M_{\delta}>x\right)=o\left(\overline{F_{\mathrm{e}}^{*}}(x)\right)=o\left(\overline{F_{\mathrm{e}}}(x)\right) .
$$


By Lemma 2.4(ii) and the local uniformity of the convergence in relation (1.2), it holds, for arbitrarily fixed $k \geq 1$, that

$$
\begin{aligned}
\int_{k \mu(1-d) \delta}^{x} \overline{F_{\mathrm{e}}}(x-y) \operatorname{Pr}\left(M_{\delta} \in \mathrm{d} y\right) & \leq\left(\int_{0-}^{\infty}-\int_{0-}^{k \mu(1-d) \delta}\right) \overline{F_{\mathrm{e}}}(x-y) \operatorname{Pr}\left(M_{\delta} \in \mathrm{d} y\right) \\
& \sim \overline{F_{\mathrm{e}}}(x) \mathrm{E} \exp \left(\gamma M_{\delta}\right) \mathbf{1}_{\left\{M_{\delta}>k \mu(1-d) \delta\right\}},
\end{aligned}
$$

where the finiteness of $\operatorname{Exp}\left(\gamma M_{\delta}\right)$ is guaranteed by (4.2). Moreover, by Lemma 2.1,

$$
\int_{x}^{\infty}(y-x) \operatorname{Pr}\left(M_{\delta} \in \mathrm{d} y\right)=\int_{x}^{\infty} \operatorname{Pr}\left(M_{\delta}>y\right) \mathrm{d} y=o(1) \int_{x}^{\infty} \overline{F_{\mathrm{e}}}(y) \mathrm{d} y=o\left(\overline{F_{\mathrm{e}}}(x)\right) .
$$

Substituting (4.2)-(4.4) into (4.1) yields the desired assertion.

Proposition 4.2. Under the conditions of Theorem 1.1(ii) for the case in which $\gamma>0$, relation (3.6) holds for each $k=2,3, \ldots$..

Proof. By Lemma 2.1, $F_{\mathrm{e}} \in \delta(\gamma)$ for some $\gamma>0$ implies that $F \in \delta(\gamma)$. When $1=n<$ $m \leq k$, for arbitrarily fixed $D>0$, we have

$$
\begin{aligned}
\operatorname{Pr}\left(Y_{1}\right. & \left.>x, Y_{m}-S_{m-1}>x\right) \\
& \leq \operatorname{Pr}\left(-S_{m-1}>x-D\right)+\operatorname{Pr}\left(Y_{1}>x, Y_{m}-S_{m-1}>x,-S_{m-1} \leq x-D\right) \\
& \leq \operatorname{Pr}\left(-S_{m-1}>x-D\right)+\operatorname{Pr}\left(Y_{1}>x, Y_{m}>D\right) .
\end{aligned}
$$

By Lemma 2.4(ii),

$$
\operatorname{Pr}\left(-S_{m-1}>x-D\right)=o(\bar{F}(x-D))=o(\bar{F}(x)) .
$$

Substitute this into (4.5), then note that $D$ can be arbitrarily large. It follows that

$$
\operatorname{Pr}\left(Y_{1}>x, Y_{m}-S_{m-1}>x\right)=o(\bar{F}(x))=o\left(\overline{F_{\mathrm{e}}}(x)\right) .
$$

Similarly, when $1<n<m \leq k$, for arbitrarily fixed $D>0$,

$$
\begin{aligned}
& \operatorname{Pr}\left(Y_{n}-S_{n-1}>x, Y_{m}-S_{m-1}>x\right) \\
& \leq \operatorname{Pr}\left(-S_{n-1}>x-D\right) \\
& \quad+\int_{-\infty}^{x-D} \operatorname{Pr}\left(Y_{n}>x-y, Y_{m}-S_{n, m-1}>x-y\right) \operatorname{Pr}\left(-S_{n-1} \in \mathrm{d} y\right),
\end{aligned}
$$

where $S_{n, m-1}=\sum_{i=n}^{m-1} X_{i}$, as before. By (4.6), for arbitrarily small $\varepsilon>0$, choose $D>0$ such that

$$
\operatorname{Pr}\left(Y_{n}>x, Y_{m}-S_{n, m-1}>x\right) \leq \varepsilon \overline{F_{\mathrm{e}}}(x)
$$

for $1<n<m \leq k$ and all $x \geq D$. Using this inequality and Lemma 2.4(ii), we obtain

$$
\begin{aligned}
\operatorname{Pr}\left(Y_{n}-S_{n-1}>x, Y_{m}-S_{m-1}>x\right) & \leq o\left(\overline{F_{\mathrm{e}}}(x)\right)+\varepsilon \int_{-\infty}^{x-D} \overline{F_{\mathrm{e}}}(x-y) \operatorname{Pr}\left(-S_{n-1} \in \mathrm{d} y\right) \\
& \lesssim \varepsilon \operatorname{E} \exp \left(-\gamma S_{n-1}\right) \overline{F_{\mathrm{e}}}(x) .
\end{aligned}
$$

This proves that

$$
\operatorname{Pr}\left(Y_{n}-S_{n-1}>x, Y_{m}-S_{m-1}>x\right)=o\left(\overline{F_{\mathrm{e}}}(x)\right) .
$$

A combination of (4.6) and (4.7) gives (3.6). 


\subsection{Proof of Theorem 1.1(ii)}

The proof for the case in which $\gamma>0$ can be given by copying the proof of Theorem 1.1(i) for the case in which $\gamma>0$ with only the modifications that we use in Lemma 2.4(ii) and Propositions 4.1 and 4.2 instead of Lemma 2.4(i) and Propositions 3.1 and 3.2.

We consider the case in which $\gamma=0$, and we aim at relation (3.15). The proof of the asymptotic lower bound is the same as that in Theorem 1.1(i). The proof of the asymptotic upper bound can be found in Palmowski and Zwart (2007). Nevertheless, for the sake of self-containedness, we copy their proof here.

For an arbitrarily large but fixed number $y>0$, define

$$
Z=(-X) \mathbf{1}_{\{(-X) \vee Y \leq y\}}+((-X) \vee Y) \mathbf{1}_{\{(-X) \vee Y>y\}} .
$$

Clearly, $Z=Z(y)$ converges to $-X$ almost surely as $y \rightarrow \infty$ and $\mathrm{E} Z<0$ for all large $y$. Moreover, it is easy to see that the relation $\operatorname{Pr}(Z>x) \sim \bar{F}(x)$ holds for arbitrarily fixed $y$. Define $Z_{n}$ in a similar way in terms of $X_{n}$ and $Y_{n}, n=1,2, \ldots$, so that $\left\{Z_{n}, n=1,2, \ldots\right\}$ forms a sequence of i.i.d. copies of $Z$. Then, we arrive at the following key inequality of Palmowski and Zwart (2007):

$$
M=\sup _{n \geq 1}\left(Y_{n}-S_{n-1}\right) \leq \sup _{n \geq 1} \sum_{i=1}^{n-1} Z_{i}+y .
$$

Therefore, by Theorem 2(B) of Veraverbeke (1977),

$$
\operatorname{Pr}(M>x) \leq \operatorname{Pr}\left(\sup _{n \geq 1} \sum_{i=1}^{n-1} Z_{i}>x-y\right) \sim-\frac{1}{\mathrm{E} Z} \int_{x-y}^{\infty} \bar{F}(u) \mathrm{d} u .
$$

Since $F_{\mathrm{e}} \in \delta$ and $y$ can be arbitrarily large, it follows that

$$
\operatorname{Pr}(M>x) \lesssim \frac{1}{\mu} \int_{x}^{\infty} \bar{F}(u) \mathrm{d} u
$$

\section{Acknowledgements}

The authors would like to thank the anonymous referee and the Editor for their insightful comments, which have helped us extend the scope of this study. The third author acknowledges the support of the National Natural Science Foundation of China (grant number 70501028), the Beijing Sustentation Fund for Elitist (grant number 20071D1600800421), the National Key Technologies R\&D Program (grant number 2006BAJ07B01), and the Research Grant of Renmin University of China (grant number 08XNA001).

\section{References}

Araman, V. F. And Glynn, P. W. (2006). Tail asymptotics for the maximum of perturbed random walk. Ann. Appl. Prob. 16, 1411-1431.

Asmussen, S., Schmidli, H. AND Schmidt, V. (1999). Tail probabilities for non-standard risk and queueing processes with subexponential jumps. Adv. Appl. Prob. 31, 422-447.

Bingham, N. H., Goldie, C. M. And Teugels, J. L. (1987). Regular Variation (Encyclopaedia Math Appl. 27). Cambridge University Press.

Pakes, A. G. (2004). Convolution equivalence and infinite divisibility. J. Appl. Prob. 41, 407-424.

Palmowski, Z. And Zwart, B. (2007). Tail asymptotics of the supremum of a regenerative process. J. Appl. Prob. 44, 349-365. 
Robert, C. Y. (2005). Asymptotic probabilities of an exceedance over renewal thresholds with an application to risk theory. J. Appl. Prob. 42, 153-162.

Rogozin, B. A. And Sgibnev, M. S. (1999). Banach algebras of measures on the line with given asymptotics of distributions at infinity. Siberian Math. J. 40, 565-576.

Su, C. And TANG, Q. (2003). Characterizations on heavy-tailed distributions by means of hazard rate. Acta Math. Appl. Sin. Engl. Ser. 19, 135-142.

TANG, Q. (2007). The overshoot of a random walk with negative drift. Statist. Prob. Lett. 77, 158-165.

Veraverbeke, N. (1977). Asymptotic behaviour of Wiener-Hopf factors of a random walk. Stoch. Process. Appl. 5, 27-37.

\section{Note added in proof}

During the proofreading stage Professors Zbigniew Palmowski and BertZwart kindly pointed out to us that our Theorem 1.1(i) for $\gamma>0$ and Theorem 1.1(ii) have been covered by Theorems 1 and 2 of Palmowski and Zwart (2007). 